\title{
Simulation of Electrohydrodynamic Jet Flow in Dielectric Fluids
}

\author{
H. Sugiyama ${ }^{1}$, H. Ogura ${ }^{2}$, T. Shiojima ${ }^{2}$ and Y. Otsubo ${ }^{2 \dagger}$ \\ ${ }^{I}$ Division of Diversity and Fractal Science, Graduate School of Science and Technology, Chiba University, Yayoi- \\ cho 1-33, Inage-ku, Chiba-shi, Chiba, 263-8522 Japan \\ 2 Department of Urban Environment Systems, Graduate School of Engineering, Chiba University, Yayoi-cho 1-33, \\ Inage-ku, Chiba-shi, 263-8522, Japan \\ †Corresponding Author Email: yas.otsubo@faculty.chiba-u.jp
}

(Received December 12, 2011; accepted January 12, 2013)

\begin{abstract}
When a dielectric fluid is exposed to a high electric field $\left(>1 \mathrm{kVmm}^{-1}\right)$, electric forces are generated due to the nonuniformity of electric conductivity and dielectric constant. The electric body forces often produce complex and macroscopic flow such as convection, turbulent and chaotic flow. The secondary flow induced in high electric fields is well known as electrohydrodynamic (EHD) effects. According to previous EHD experiments and numerical simulation in DC fields, the velocity of flow has been reported to be of the order of $10^{-2} \mathrm{~ms}^{-1}$ in electric fields of several $\mathrm{kVmm}^{-1}$. However, on the application of high DC electric fields to some dielectric oils, a fluid jet with a velocity of about $1 \mathrm{~ms}^{-1}$ can be created from the positive electrode as a bulk flow. In this study, the numerical simulation of EHD jet is carried out from the engineering aspects. The high speed jet flow is theoretically reproduced, and the obtained flow patterns are compared with the experimental results under the conditions of simple electrode allocations.
\end{abstract}

Keywords: Electrohydrodynamics, Simulation, Jet flow, Dielectric fluids.

\section{NOMENCLATURE}

$\begin{array}{ll}E & \text { electric field intensity, } \mathrm{Vm}^{-1} \\ f & \text { force, } \mathrm{N} \\ L & \text { characteristic length, } \mathrm{m} \\ p & \text { pressure, } \mathrm{Pa} \\ t & \text { time, } \mathrm{s} \\ U & \text { characteristic velocity, } \mathrm{ms}^{-1} \\ u & \text { velocity, } \mathrm{ms}^{-1} \\ V_{0} & \text { characteristic field potential, } \mathrm{V} \\ \varepsilon & \text { permittivity, } \mathrm{F} \mathrm{m}^{-1} \\ \phi & \text { electric field potential, } \mathrm{V} \\ \mu & \text { viscosity, } \mathrm{Pas}^{2} \\ \mu_{e} & \text { mobility, } \mathrm{m}^{2} \mathrm{~V}^{-1} \mathrm{~s}^{-1} \\ \rho & \text { density, } \mathrm{kgm}^{-3} \\ \rho_{e} & \text { charge density, } \mathrm{Cm}^{-3}\end{array}$

\section{INTRODUCTION}

Dielectric fluids are characterized by very low values of the conductivity $\left(<10^{-8} \mathrm{Sm}^{-1}\right)$ and commonly regarded as insulating oils in industries. When dielectric fluids are subjected to the high electric field $\left(>1 \mathrm{kVmm}^{-1}\right)$, electric body forces are

$$
\begin{aligned}
& \rho_{e 0} \quad \text { characteristic charge density, } \mathrm{Cm}^{-3} \\
& \sigma \quad \text { electric conductivity, } \mathrm{Sm}^{-1} \\
& \text { Dimensionless numbers } \\
& C_{\text {oul }}=\frac{\rho_{e 0} V_{0}}{\rho U^{2}} \\
& E_{\text {lec }}=\frac{\sigma V_{0}}{\rho_{e 0} L U} \\
& P_{\text {erm }}=\frac{L^{2} \rho_{e 0}}{\varepsilon V_{0}} \\
& R_{e}=\frac{\rho L U}{\mu} \\
& V_{m o b}=\frac{\mu_{e} V_{0}}{L U}
\end{aligned}
$$

generated due to the non-uniformity of electric conductivity and dielectric constant. Under some conditions, macroscopic motions such as convection, turbulence, and chaos are induced in the systems. The secondary motions of fluid, which are produced in high electric fields, is known as electrohydrodynamic (EHD) effects. (Castellanos 1991, Maekawa et al. 1992, Suzuki 
1985, Worraker and Richardson, 1981). The EHD effects are extensively studied from theoretical and experimental points. Recently the application of EHD flow to mechanical devices has received increasing attention. Zahn and Reddy (2006) have analyzed the micromixing process due to EHD instability for techniques of separation of DNA from other cellular components. For electronic cooling, the EHD micropumps have been extensively studied by Darabi $e t$ al. (2001, 2002, 2005, 2006). In the field of colloid science, the EHD flow of suspensions, which contain nanoparticles, has been accepted as the key technology of direct writing of nanoparticles for nanoprinting and formation nanostructures (Rocks et al. 2007, Wang et al. 2007). Also, the EHD flow can be used for formation of fine fibers and this method is called electrospinning (Skotak and Larsen 2006, Park et al. 2008). In DC fields, the Coulomb force acting on a space charge dominates the dielectrophoretic force and often causes the EHD instability. According to the numerical simulation and EHD experiments in DC fields, the velocity of the flow has been reported to be of the order of $10^{-2} \mathrm{~ms}^{-1}$ in electric fields of several $\mathrm{kVmm}^{-1}$ (Denat et al. 1979, Yasufuku et al. 1979, Haga et al. 1995).

In previous papers, we have found that on the application of high DC fields to insulating oils, a fluid jet with a velocity of about $1 \mathrm{~ms}^{-1}$ is created from the positive electrode. In this process, electric energy is directly converted to kinetic energy of the fluid. Since the high energy density can be generated, the EHD jet is very attractive in applications to mechanical elements. By controlling the velocity and the direction of EHD jet, the authors have developed three kinds of new fluid devices. The first devices are the dielectric fluid motors (Otsubo and Edamura 1997), which consist of vane wheel, cup, wire electrodes, and working fluid. Several sets of wire electrodes are arranged around the inner wall of cylinder. The rotational direction is regulated by the polarity because the flow of working fluid is induced in the direction from the positive to negative electrodes. By modification of geometry and electrode design, various types of motors have been developed (Yokota et al. 2001a, b).

The second devices are electrorheological (ER) devices. The ER effect is defined as a rapid and reversible change in viscosity of fluids on the application or removal of electric fields. Typical ER fluids are suspensions of polarizable particles dispersed in oils. But when subjected to high electric fields in electrodes with flocked fabrics, the pure oils without particles can cause the increase in viscosity (Otsubo and Edamura 1998, 1999). The most interesting feature is that the viscosity behavior is converted from Newtonian to shear-thinning profiles and the system becomes a viscoelastic fluid due to striking elastic effects. The rapid and large-scale convection is generated between the tips of fibers and plate electrode. Since the additional energy is required to change the periodic patterns of EHD convection by forced shear, the ER effect demonstrated by the modification of electrode with flocked fabrics can be attributed to a combined effect of EHD convection and external shear.
The third devices are inkjet nozzles (Edamura and Otsubo 2004). The electric fields acting on space charges produce not only the body forces in the bulk, but also the extra stresses across the interfaces. If the sufficiently high normal stresses are exerted, the continuous evolution of free surface from the nozzle exit can take place. By the use of a needle electrode, the continuous liquid jet can be generated from the nozzle exit. This can provide the feasibility of new basic technology for inkjet printing. The developed models are very attractive as the elements of new fluid devices and have great potential in the field of fluid machine technology. However, the mechanism of jet flow generated in electric fields is not clearly explained. For improvement of device performance, it is essential to understand the EHD jet mechanism as a function of fluid properties. In the present paper, the large-scale EHD convection is experimentally examined in simplified conditions and the obtained results will be quantitatively analyzed through numerical simulation.

\section{EHD FlOW PATTERNS GENERATED AMONG MUlTiple EleCTRODES}

\subsection{Materials and Methods}

The sample fluid was dibutyl decanedioate, whose density and viscosity were $0.936 \times 10^{3} \mathrm{kgm}^{-3}$ and 10.1 $\mathrm{mPas}$ at $25^{\circ} \mathrm{C}$, respectively. The electrical conductivity was $1.03 \times 10^{-9} \mathrm{Sm}^{-1}$ at $1 \mathrm{kVmm}^{-1}$ and $1.35 \times 10^{-9} \mathrm{Sm}^{-1}$ at $2.0 \mathrm{kVmm}^{-1}$. A very small amount of silica particles were dispersed as tracers for image analysis.

The fluid was placed in a petri dish at a thickness of 15 $\mathrm{mm}$, in which several electrodes made of copper wire with a thickness of $0.1 \mathrm{~mm}$ were vertically immersed at a depth of $1.0 \mathrm{~mm}$. A DC voltage of $4.0 \mathrm{kV}$ was applied to the positive electrodes and the negative electrodes were connected to the ground $(0 \mathrm{~V})$. The macroscopic flow electrically induced at the liquid surface was recorded through a high-speed videocamera (FASTCAM-Net 1000C produced by Photron). The velocity profiles were calculated from the images taken at an interval of $1 \mathrm{~ms}$ by the use of Pc-Vector obtained from Ohyoh Keisoku Kenkyusho, Inc. In electric fields generated by different geometrical arrangements of electrodes, the flow patterns were determined after the steady flow states were established.

\subsection{Experimental Results}

Figure 1 shows the effect of electrode arrangement on the velocity profiles. Each arrow shows the direction and magnitude of flow at the point where the line starts. When the high voltage is applied to the sample fluid, the high-speed jet flow is generated around positive electrodes and the patterns of flow fields become steady in several seconds. In arrangement (A), one set of electrodes is immersed in the fluid at a separation of 20 $\mathrm{mm}$. The maximum velocity is attained near the positive electrode in the direction to negative electrode. The value is about $80 \mathrm{mms}^{-1}$ on the line between two electrodes. In addition, the vortical flows with low velocity are confirmed behind the positive electrode. The jet flow is observed at voltages above $1.5 \mathrm{kV}$. 


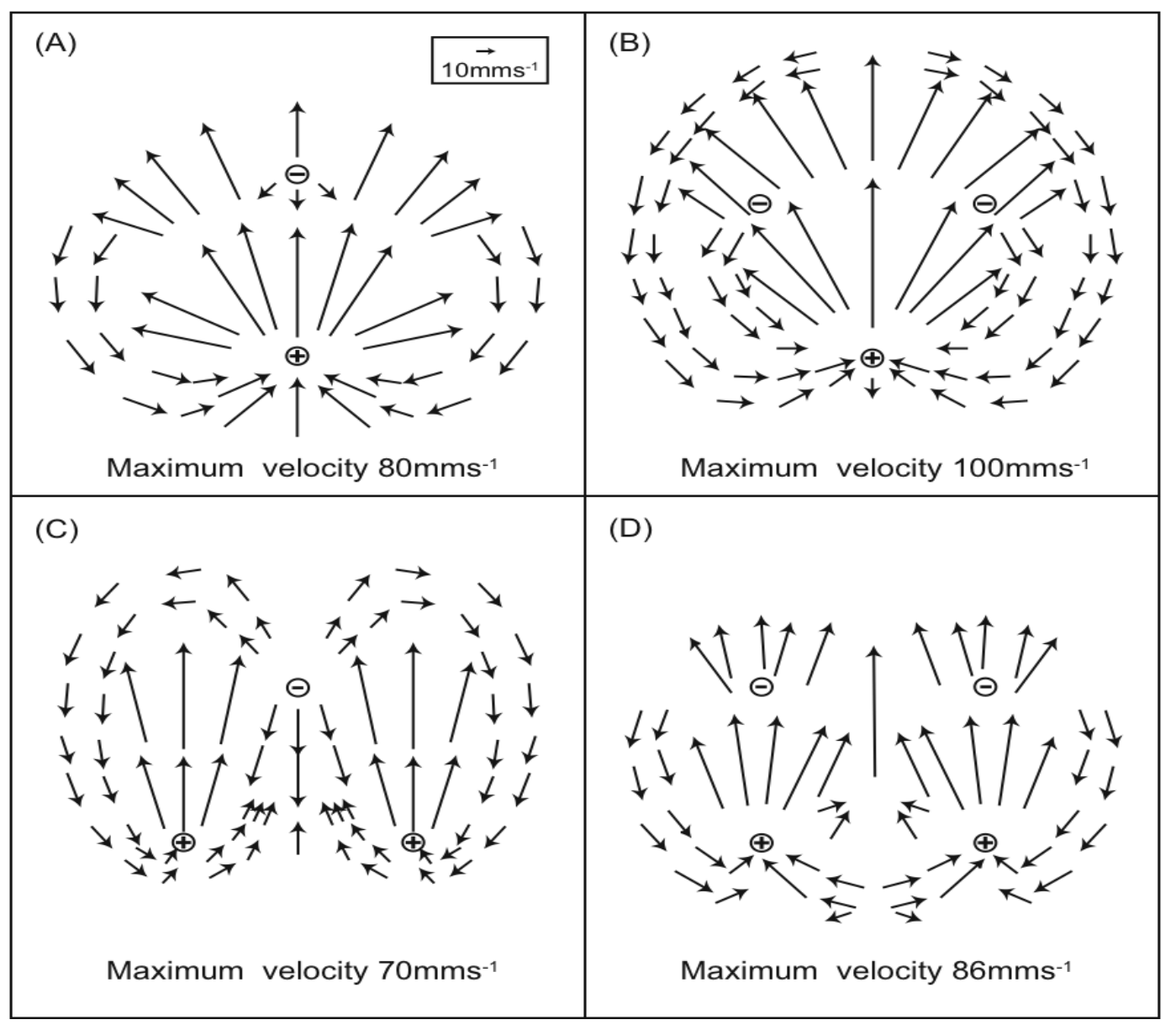

Fig. 1. Macroscopic movement of the EHD jet in different electrode arrangements.

Hence there exists a critical voltage that must be overcome for continuous formation of jet flow. Beyond the critical voltage, the velocity increases with increasing applied voltage.

In arrangements $\mathrm{B}$ and $\mathrm{C}$, three electrodes are set in the form of equilateral triangle at an electrode separation of $20 \mathrm{~mm}$, whereas the polarity is changed. One electrode is positive and two electrodes are negative in arrangement (B). From the results in arrangement (A), it is easily expected that the flow is generated from positive to two negative electrodes, that is, the flow diverges in two directions. However, the main flow passes through the gap of two negative electrodes. Since the maximum velocity is about $100 \mathrm{mms}^{-1}$ near the positive electrode, the velocity profile is very similar to that obtained in arrangement (A). The addition of negative electrode does not strongly affect the velocity profile. On the other hand, the velocity profile is quite different in arrangement (C), in which two electrodes are positive and one electrode is negative. The most significant aspect is that the jet flow is induced from the negative electrode, although the main flow with maximum velocity is issued from the positive electrodes. It looks likely that the jet flows induced from the positive and negative electrodes are canceled in the center region of triangle. As a result, two strong parallel flows appear, the maximum velocity of which is $70 \mathrm{mms}^{-1}$, as if the negative electrode serves to repel the flow from the positive electrodes.

In arrangement (D), two sets of electrodes are placed in parallel to form a square at an electrode separation of $20 \mathrm{~mm}$. Since the parallel jet flows are induced, the overall velocity profile can be explained by a superposition of flows observed in arrangement (A). But an interesting point is that the maximum velocity of $86 \mathrm{mms}^{-1}$ is obtained in the center region of square. Presumably the EHD jet flows are enhanced by two negative electrodes in a similar manner that the accelerated flow passes through the gap of two negative electrodes in arrangement (B).

The velocity profiles of EHD jet in different electrode arrangements can be summarized as follows: The main jet is generated from positive to negative electrodes. Although the weak flow from negative electrodes is also observed, it is overwhelmed by the main flow. In the triangle and square arrangements, the vector of maximum velocity does not lie on the line connecting positive and negative electrodes. Because of cooperative motion of fluid elements, the velocity profiles are strikingly influenced by the geometry of electrode arrangement. 


\section{PAPER Format NuMERICAL Simulation Of EHD Flow Profiles}

\subsection{Basic Equations}

The basic equations governing the unsteady incompressible viscous EHD flow are expressed as follows:

(a) Continuity equation

$\nabla \cdot u=0$

(b) Momentum equation

$\rho \frac{\partial u}{\partial \mathrm{t}}+\rho u \cdot \nabla u=-\nabla p+\mu \nabla^{2} u+f$

where $f=-\rho_{e} \nabla \phi$

(c) Charge transport equation

$\frac{\partial \rho_{e}}{\partial \mathrm{t}}+u \cdot \nabla \rho_{e}=\left(\sigma+\rho_{e} \mu_{e}\right) \nabla^{2} \phi$

(d) Electrostatic equation

$\nabla \cdot E=\frac{\rho_{e}}{\varepsilon}$

where $u$ is the velocity $\left[\mathrm{ms}^{-1}\right], t$ is the time $[\mathrm{s}], p$ is the pressure $[\mathrm{Pa}], \rho$ is the density $\left[\mathrm{kgm}^{-3}\right], \rho_{e}$ is the charge density $\left[\mathrm{Cm}^{-3}\right], \mu$ is the viscosity $[\mathrm{Pas}], \mu_{e}$ is the mobility $\left[\mathrm{m}^{2} \mathrm{~V}^{-1} \mathrm{~s}^{-1}\right], \varepsilon$ is the permittivity $\left[\mathrm{Fm}^{-1}\right], \sigma$ is the electric conductivity $\left[\mathrm{Sm}^{-1}\right]$, and $\phi$ is the electric field potential [V].

These equations can be rewritten by the use of five dimensionless numbers, which are defined as

$$
\begin{aligned}
& R_{e}=\frac{\rho L U}{\mu} \\
& C_{\text {oul }}=\frac{\rho_{e 0} V_{0}}{\rho U^{2}} \\
& E_{\text {lec }}=\frac{\sigma V_{0}}{\rho_{e 0} L U} \\
& V_{\text {mob }}=\frac{\mu_{e} V_{0}}{L U} \\
& P_{\text {erm }}=\frac{L^{2} \rho_{e 0}}{\varepsilon V_{0}}
\end{aligned}
$$

where the length $L$, velocity $U$, charge density $\rho_{e 0}$ and electric potential $V_{0}$ are the characteristic vales for nondimensionalization.

\subsection{Numerical Analysis}

Equations (1) and (2) are solved by the SMAC scheme (Dodge 1977, Mckee et al. 2008), in which the forward Euler method is applied to convective and viscous terms, and the implicit Euler method to the pressure term, through the time-splitting procedure. To assure the numerical stability and accuracy, the implicit Crank-Nicolson method and the second order AdamsBashforth method are used for viscous term and body force, respectively. The calculation results of Eq.(3) are often unstable for high Reynolds number $R_{e}$, the upwind-difference scheme is applied to the convection derivative terms and Chakravarthy-Osher second order TVD scheme to flux calculation (Chakravathy and Osher 1985). The equations are solved by the finite difference method by the use of an irregular rectangle grid and the SOR method is applied for solving a linear system of equations.

The geometry is a square with a length of $20 \mathrm{~cm}$. The boundary conditions are

(1) No slip condition on the solid wall is prescribed.

(2) The natural (Neumann) conditions are imposed for the electric potential, pressure, electric charge on the solid wall.

The computational procedures for simulation of EHD flow profile are as follow:

(1) Set up the grid, physical parameter and boundary conditions.

(2) Input the starting values. Usually all variables are predetermined to be zero.

(3) Compute the potentials of the electric field by Eq. (4).

(4) Predict the velocity and solve the vector potential by the linear equations.

(5) New pressure are calculated.

(6) Compute the velocity to satisfy the continuity condition.

(7) Compute the transportations of the electric charge by Eq. (3)

By repeating the procedures from (2) to (7) steps, the stable and accurate flow patterns are obtained.

\subsection{Results of Simulation}

Figure 2 shows the contours of electric potential at an electrode voltage of about $4.0 \mathrm{kV}$ in different electrode arrangements. The voltage drop around the negative electrodes is very steep. The potential patterns are easily understood from the geometry of electrode arrangement. Although the charge distribution patterns are not shown, it has been confirmed that the charges are concentrated in the vicinity of positive electrodes. Because of low electric current, the charge density is suddenly decreased in the area apart from the positive electrodes.

Figure 3 shows the velocity fields determined under the same conditions as in Fig. 1. The change in velocity is shown by the color of arrow, that is, the velocity increases in the order of blue $\rightarrow$ green $\rightarrow$ red. In arrangement (A), the main jet flows issued from the positive electrode and the flow patterns are very similar to the experimental results. Moreover, the vertical flows drawn to the positive electrode in the rear region are reproduced in good agreement with the experimental results. In simulation, all flows are generated by the motion of electric charges. Therefore, the intrinsic mechanism of high-speed EHD flows may arise from the convective transport of the charge carriers or convective current. However, it was not successful in the present programs to predict the weak flows generated from negative electrode, which were experimentally observed. The maximum velocity obtained in the steady-state simulation is $37.9 \mathrm{mms}^{-1}$. The low value compared with experimental data may be 
caused by the effects of impurity, surface tension, EHD of surrounding air, electric double layer formed around the electrodes, and heat convection. These effects are neglected in the simulation. The jet flow with a velocity beyond $1.0 \mathrm{~ms}^{-1}$ is established at the voltages above $20 \mathrm{kV}$ in this program.

In arrangement (B), the flow does not diverge in two directions of negative electrodes, but the main strong flow is constructed between two negative electrodes. This implies that the strongest electric field lies along the line between the positive electrode and the middle point of two negative electrodes. Because of higher fled strength, the flow in arrangement (B) is faster than that in (A). In arrangement $(\mathrm{C})$, the flow is not directed to the negative electrode, but two strong parallel flows are generated from two positive electrodes. The stagnation region appears in the vicinity of negative electrode. Avoiding this region, two jet flows are generated, the velocity of which is lower than that in arrangement (A). Also, in arrangement (D), the flow profiles obtained by the experiments and simulations are very similar. As expected from Fig. 1, it is shown that the addition of negative electrode to arrangement (C) does not significantly influence the EHD flow patterns in simulation. As a result, the effects of polarity on the flow patterns are well reproduced by the simulation.

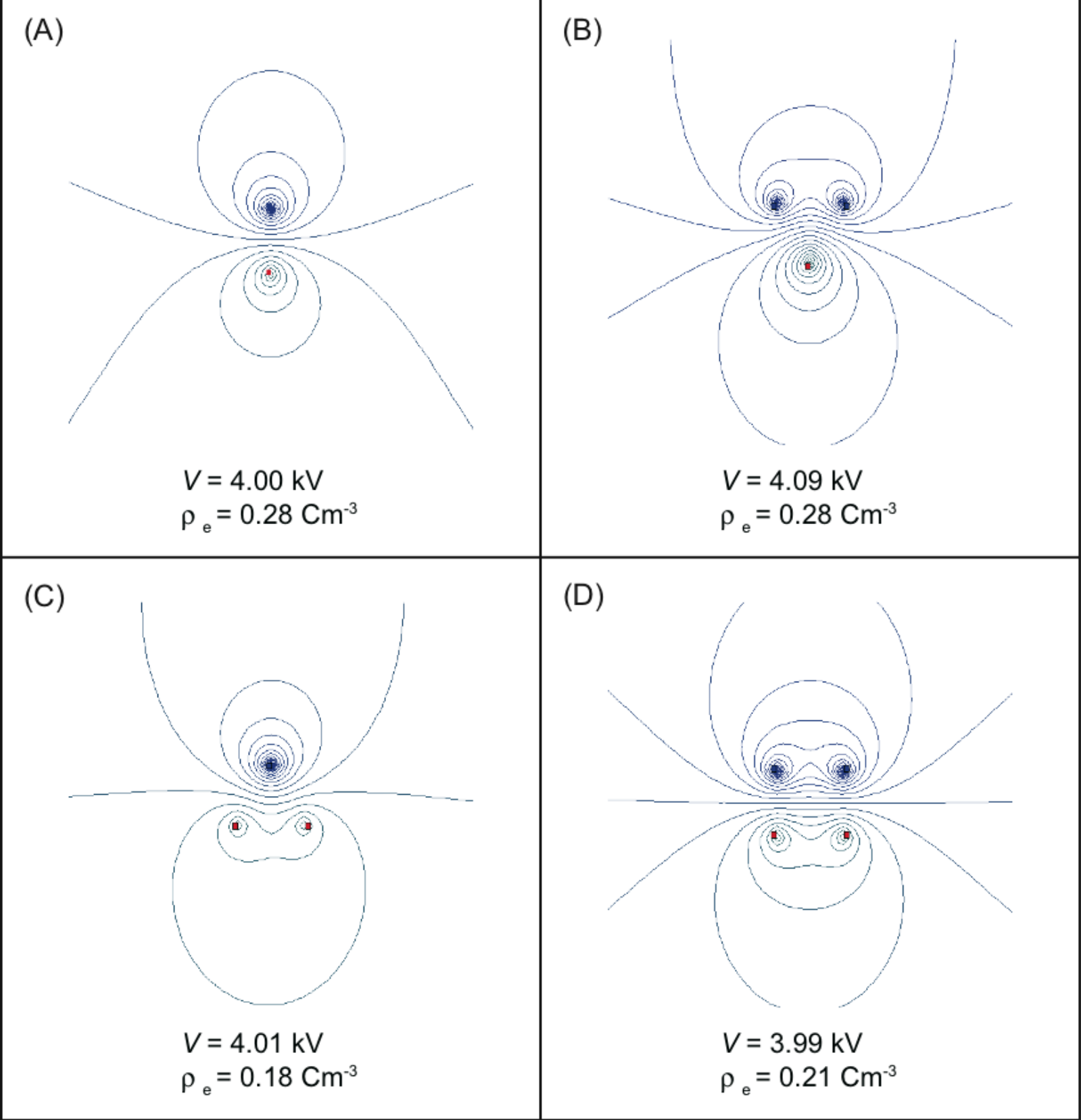

Fig. 2. Contour lines of voltage in different electrode arrangements. 


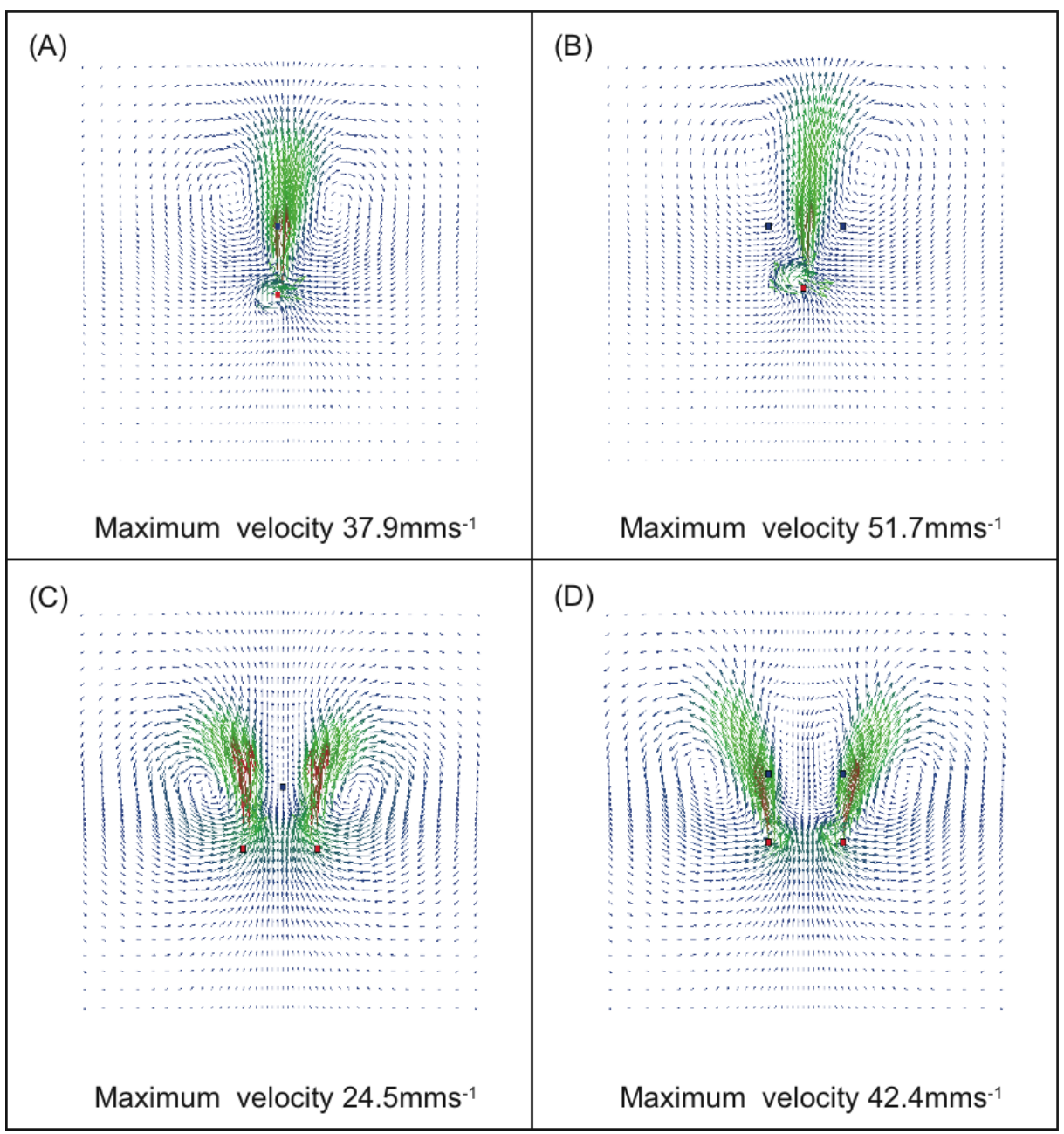

Fig. 3. Patterns of velocity fields at $t=3.0 \mathrm{~s}$.

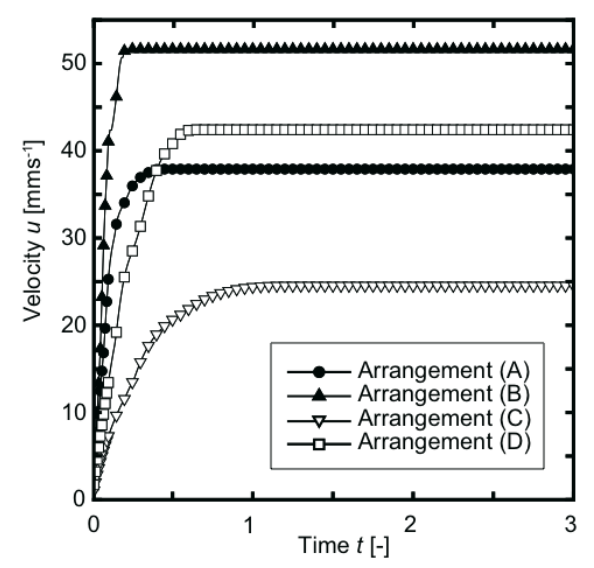

(A) Velocity

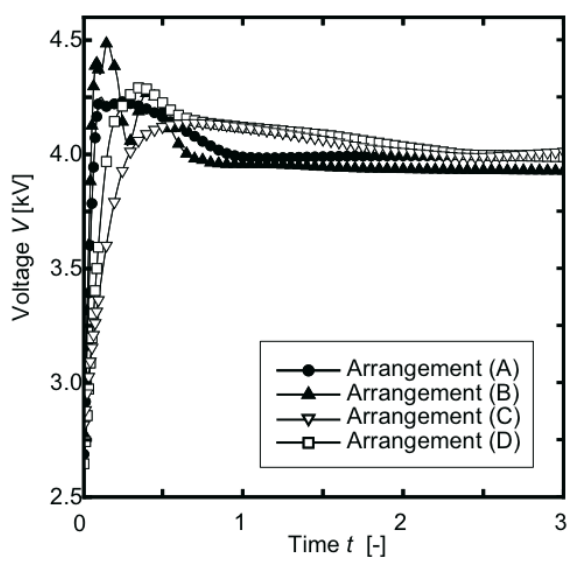

(B) Voltage

Fig. 4. Time dependence of velocity and voltage after the electrification.

Figures 4(A) and (B) show the time dependence of maximum values of velocity and voltage, respectively, after the electrification in different arrangements of electrodes. The velocity increases with time and reaches the saturation which depends on the electrode arrangement, while the voltage shows a sudden increase 
and gradually decreases to the constant values. In the simulation, the voltage of positive electrodes is controlled to converge to the constant value of $4.0 \mathrm{kV}$, irrespective of geometry of arrangement. The overdose of charges at the beginning of electrification may be responsible for the appearance of peak in timedependent curve of voltage. From the time dependence of velocity and voltage, it is established that the patterns shown in Fig. 3 give the distribution of velocity in the stable states.

\section{Conclusion}

On the application of high DC fields to insulating oils, a high-speed jet flow is created from the positive electrode. The EHD flow patterns generated among multiple electrodes are changed with geometry of electrode arrangement. To reproduce the experimental results, the numerical analysis is carried out by the use of continuity equation, momentum equation, charge transport equation, and electrostatic equation. The flow profiles obtained by the experiments and simulations are very similar. The numerical analysis is successful in quantitative explanation of the experimental results.

\section{REFERENCES}

Castellanos, A. (1991). Coulomb-driven convection in electrohydrodynamics. IEEE Transactions on Electrical Insulation, 26(6), 1201-1215.

Chakravathy, S. R. and S. Osher (1985). A new class of high accuracy TVD schemes for hyperbolic conservation law. American Institute of Aeronautics and Astronautics Paper (AIAA) 85 -0363, AIAA, New York, NY.

Darabi, J. and C. Rhodes (2006). CFD modeling of an ion-drag pump, Sensor and Actuators A: Physics, 127(1), 94-103.

Darabi, J. and H. Wang (2005). Development of an electrohydrodynamic injection micropump and its potential application in pumping fluids in cryogenic cooling systems, Journal of Microelectromechanical Systems, 14(4), 747-755.

Darabi, J., M. Rada, M. M. Ohadi and J. Lawler (2002). Disign, fabrication, and testing of an electrohydrodynamic ion-drag micropump. Journal of Microelectromechnical Systems, 11(6), 684-690.

Darabi, J., M. M. Ohadi and D. DeVoe (2001). An electrohydrodynamic polarization micropump for electronic cooling, Journal of Microelectromechanical Systems, 10(1), 98-106.

Denat, A., B. Gosse and J. P. Gosse (1979). Ion injections in hydrocarbons. Journal of Electrostatics, 7, 205-225.

Dodge P. R. (1977). Numerical method for 2D and 3D viscous flow. American Institute of Aeronautics and Astronautics Journal (AIAA), 15(7), 961-965.
Edamura, K. and Y. Otsubo (2004). A continuous inkjet device on the basis of electrohydrodynamic mechanism. Journal of Imaging Science and Technology, 48(2), 148-152.

Haga, M., T. Maekawa, K. Kuwahara, A. Ohara, K. Kawasaki, T. Harada, S. Yoda and T. Nakamura, (1995). Effect of electric field on Marangoni convection under microgravity. Journal of the Japan Society of Microgravity Application, 12 ,1926.

Maekawa, T., K. Abe and I. Tanasawa (1992). Onset of natural convection under an electric field. International Journal of Heat and Mass Transfer, 35(3), 613-621.

McKee, S., M. F. Tome, V. G. Ferreira, J. A. Cuminato, A. Castelo, F. S. Sousa and N. Mangiavacchi (2008). The MAC method. Computers and Fluids, 37(8), 907-930

Otsubo, Y. and K. Edamura (1999). Electric effect on the rheology of insulating oils in electrodes with flocked fabrics. Rheologica Acta, 38(2), 137-144.

Otsubo, Y. and K. Edamura (1998). Viscoelasticity of a dielectric fluid in nonuniform electric fields generated by electrodes with flocked fabrics. Rheologica Acta, 37(5), 500-507.

Otsubo, Y. and K. Edamura (1997). Dielectric fluid motors. Applied Physics Letter, 71(3), 318-320.

Park, C. H., K. H. Kim, J. C. Lee and J. Lee (2008). Insitu nanofabrication via electrohydrodynamic jetting of countercharged nozzles. Polymer Bulletin, 61(4), 521-528.

Rocks, S. A., D. Wang, D. Sun, S. N. Jayasinghe, M. J. Edirisinghe and R. A. Dorey (2007). Direct writing of lead zirconate titanate piezoelectric structures by electrohydrodynamic atomization. Journal of Electroceramics, 19(4), 287-293.

Skotak, M. and G. Larsen (2006). Solution chemistry control to make well defined submicron continuous fibres by electrospinning: the $\left(\mathrm{CH}_{3} \mathrm{CH}_{2} \mathrm{CH}_{2} \mathrm{O}\right)_{4} \mathrm{Ti} / \mathrm{AcOH} /$ poly $\quad(\mathrm{N}-$ vinylpyrolidone) system. Journal of Materials Chemistry, 16(29), 3031-3039.

Suzuki, M. (1985). Propagating transitions of Electroconvection. Physical Review A, 31, 2548-2555.

Wang, D. Z., S. N. Jayasinghe, M. J. Edirisinghe and Z. B. Luklinska (2007). Coaxial electrohydrodynamic direct writing of nano-suspensions. Journal of Nanoparticle Research, 9(5), 825-831.

Worraker, W. J. and A. T. Richardson (1981). Nonlinear electrohydrodynamic stability analysis of a thermally stabilized plane layer of dielectric liquid. Journal of Fluid Mechanics, 109, 217-237. 
H. Sugiyama et al. / JAFM, Vol. 6, No. 3, pp. 405-412, 2013.

Yasufuku, S., T. Umemura and T. Tanii (1979) Electric conduction phenomena and carrier mobility behavior in dielectric fluids, IEEE Transactions on Electrical Insulation, EI-14(1), 28-35.

Yokota, S., M. Hirata, Y. Kondoh, K. Suzumori, A. Sadamoto, Y. Otsubo and K. Edamura (2001a). Micromotor using electroconjugate fluid (fabrication of inner diameter $2 \mathrm{~mm}$ RE type ECF motor). Journal of Robotics and Mechatronics, 13, 140-145.
Yokota, S., A. Sadamoto, Y. Kondoh, Y. Otsubo and K. Edamura (2001b). A micromotor using electroconjugate fluid (ECFs) (Proposition of stator electrode type (SE-type) micro ECF motors), The Japan Society of Mechanical Engineers International Journal (JSME), C 44, 756-762.

Zahn, J. D. and V. Reddy (2006). Two phase micromixing and analysis using electrohydrodynamic instabilities. Microfluid Nanofluid, 2, 399-415. 